Cytogenetics and

Cell Genetics

\title{
13th International Chromosome Conference
}

Abstracts
September 8-12, 1998

Ancona, Italy
Guest Editor

Ettore Olmo, Ancona 


\section{Under the Patronage of}

The Commission of the European Communities Università degli Studi di Ancona

Presidenza del Consiglio regionale delle Marche

Comune di Ancona

\section{Sponsored by}

The Commission of the European Communities Presidenza del Consiglio regionale delle Marche

Comune di Ancona

Banca Popolare di Brescia (BIPOP)

TELECOM Italia

Cover illustration

Camera lucida of lampbrush chromosomes of Ambystoma drawn by Walter Fleming, 1882.

\section{KARGER}

\section{S. Karger}

Medical and Scientific Publishers

Basel $\cdot$ Freiburg $\cdot$ Paris $\cdot$ London

New York $\cdot$ New Delhi $\cdot$ Bangkok

Singapore $\cdot$ Tokyo $\cdot$ Sydney
Drug Dosage

The authors and the publisher have exerted every effort to ensure that drug selection and dosage set forth in this text are in accord with current recommendations and practice at the time of publication. However, in view of ongoing research, changes in government regulations, and the constant flow of information relating to drug therapy and drug reactions, the reader is urged to check the package insert for each drug for any change in indications and dosage and for added warnings and precautions. This is particularly important when the recommended agent is a new and/or infrequently employed drug.
All rights reserved

No part of this publication may be translated into other languages, reproduced or utilized in any form or by any means, electronic or mechanical, including photocopying, recording, microcopying, or by any information storage and retrieval system, without permission in writing from the publisher or, in the case of photocopying, direct payment of a specified fee to the Copyright Clearance Center (see 'General Information').

(C) Copyright 1998 by S. Karger AG,

P.O. Box, CH-4009 Basel (Switzerland)

Printed in Switzerland on acid-free paper by

Reinhardt Druck, Base

ISBN 3-8055-6759-6 


\section{Contents}

\section{Cytogenetics and}

Cell Genetics

\section{Final Programme}

Plenary lectures

99 Abstracts L1-L24

Chromosome structure and composition

106 Poster Abstracts P1-P39

Chromosome function

116 Poster Abstracts P40-P65

Evolutionary cytogenetics

123 Poster Abstracts P66-P114

Domestic animals cytogenetics

136 Poster Abstracts P115-P131

Cultivated plants cytogenetics

140 Poster Abstracts P132-P156

Medical and cancer cytogenetics

147 Poster Abstracts P157-P202

Mutagenesis

159 Poster Abstracts P203-P225

165 Author Index 


\section{Cytogenetics and Cell Genetics}

\section{Organizing Committee}

Ettore Olmo (Secretary General)

Marco Milani Comparetti

(Treasurer)

Adriana Canapa

Maria Luisa Caniglia

Teresa Capriglione

Vincenzo Caputo

Anna La Teana

Romina Magistrelli

Laura Mazzanti

Paola Nisi Cerioni

Gaetano Odierna

Vitaliano Paolucci

Giovanni Principato

Franca Saccucci

\section{International Advisory Committee}

M.D. Bennett (United Kingdom)

G. Bernardi (France)

A. Chandley (United Kingdom)

F. Foresti (Brasil)

P. Jauhar (U.S.A.)

W. Hennig (Germany)

R.N. Jones (United Kingdom)

H.C. Macgregor (United Kingdom)

P. Moens (Canada)

M. Puertas (Spain)

C. A. Redi (Italy)

M. Rocchi (Italy)

M. Schmid (Germany)

A. Sumner (United Kingdom)

\section{Invited Speakers}

G. Bernardi (Paris)

E. Capanna (Roma)

T. Capriglione (Napoli)

I. Chudoba (Jena)

L. de Almeida-Toledo (Sao Paulo)

J.H. de Jong (Wageningen)

B. Dutrillaux (Paris)

M.A. Ferguson-Smith (Cambridge)

B. Franco (Milano)

K. Fukui (Niigata)

H. Hameister (Ulm)

J.S. Heslop-Harrison (Norwich)

T. Ikemura (Mishima)

I. King (Aberystwyth)

N. Kleckner (Harvard)

P. Lichter (Heidelberg)

J. Loidl (Vienna)

H.C. Macgregor (Leicester)

B. McKee (Knoxville)

T. Naranjo (Madrid)

B. Nicklas (Durham)

S. Pimpinelli (Roma)

M. Puertas (Madrid)

W. Reik (Cambridge)

J. Varley (Manchester) 


\section{Final Programme}

Tuesday, September 8, 1998 (Club S. Cristiana)

Registration

21.30 Dinner

\section{Wednesday, September 9, 1998 (University, Aula Magna)}

Morning

Chairpersons: G. Levan (Göteborg, Sweden); M. Milani Comparetti (Ancona, Italy)

9.30 Welcome

10.00 Opening lecture: E. Capanna (Rome, Italy) Chromosomes yesterday: a century of chromosome studies

10.45 Coffee

11.15 Lecture 2: G. Bernardi (Paris, France) Isochores and chromosomes

11.45 Lecture 3: J.S. Heslop-Harrison (Norwich, UK) Plant genes, genomes and chromosomes

12.15 Lecture 4: N. Kleckner (Harvard, USA) Interaction between and along meiotic chromosomes

13.30 Lunch

Afternoon

Chairpersons: P. Malet (Clermont-Ferrand, France); M. Rocchi (Bari, Italy)

15.30 Lecture 5: B. Nicklas (Durham, USA) Chance and checkpoint: accurate chromosome distribution in mitosis and meiosis

16.00 Lecture 6: B. Franco (Milan, Italy) $\mathrm{Xp}$ contiguous gene syndromes: from clinical observation to disease gene identification

16.30 Lecture 7: S.B. Dutrillaux (Paris, France) Chromosomes, initiating mutations and age of cancer onset

17.00 Lecture 8: J. Varley (Manchester, UK) Genetic and cytogenetic studies in inherited cancer: Li-Fraumeni syndrome
18.00 Concert

20.00 Dinner (Club S. Cristiana)

21.30 Posters: I Session

\section{Thursday, September 10, 1998 (Club S. Cristiana)}

Morning

Visit to Urbino (with Lunch)

Afternoon

15.30 Posters: II Session

17.30 Coffee

18.00 Workshops:

Room A: Evolutionary cytogenetics

(Coordinator: C.A. Redi)

Room B: Chromosome function

(Coordinator: W. Hennig)

Room C: Cultivated plants cytogenetics

(Coordinator: J.H. de Jong)

20.30 Dinner

21.30 Posters: III Session

Friday, September 11, 1998 (Club S. Cristiana, Room A)

Morning

Chairpersons: M. Bennett (Kew, UK); P. Moens (York, Canada)

8.30 Lecture 9: P. Lichter (Heidelberg, Germany) Chromosome organisation in the interphase nucleus

9.00 Lecture 10: S. Pimpinelli (Rome, Italy) The structural and functional organisation of heterochromatin

9.30 Lecture 11: T. Ikemura (Mishima, Japan) Genome and subnuclear organisation of non B-forming DNAs in mammalian chromosomes

10.00 Lecture 12: W. Reik (Cambridge, UK) Control of IGF2 imprinting and fetal growth

\section{KARGER}

Fax+4161306 1234

E-Mail karger@karger.ch

www.karger.com

\section{(ㄷ) 1998 S. Karger AG, Basel}

Access to full text and tables of contents, including tentative ones for forthcoming issues: www.karger.com/journals/ccg/ccgcont.htm 
10.30 Coffee

11.00 Lecture 13: J. Loidl (Vienna, Austria)

Premeiotic and early meiotic nuclear organization and its possible role in meiotic homology search

11.30 Lecture 14: B. McKee (Knoxville, USA)

Pairing sites and the mechanism of meiotic pairing in Drosophila

12.00 Lecture 15: M. Puertas (Madrid, Spain) Genetic control of B chromosomes transmission in maize and rye

\subsection{Applied imaging presentation}

13.30 Lunch

Afternoon

Chairpersons: M.G. Manfredi Romanini (Pavia, Italy); M. Schmid (Würzburg, Germany)

15.30 Lecture 16: T. Naranjo (Madrid, Spain) Genome evolution in Triticeae

16.00 Lecture 17: L. de Almeida-Toledo (São Paulo, Brasil) Karyotypic evolution in neotropical freshwater fish

16.30 Lecture 18: T. Capriglione (Naples, Italy) Repetitive DNA as a tool to study the phylogeny of cold-blooded vertebrates

17.00 Lecture 19: H. Hameister (Ulm, Germany) Comparative gene mapping as a tool to reconstruct the mammalian founder karyotype

17.30 Coffee

18.00 Workshops:

Room A: Medical and cancer cytogenetics (Coordinator: L. Larizza)

Room B: Chromosome structure and composition (Coordinator: A. Leitch)

Room C: Domestic animals cytogenetics (Coordinator: L. Iannuzzi)

Room D: Mutagenesis (Coordinator: A.T. Natarajan)

20.30 Dinner

\subsection{Standing Committee meeting}

Saturday, September 12, 1998 (Club S. Cristiana, Room A)

Morning

8.30 Lecture 20: M.A. Ferguson-Smith (Cambridge, UK) Comparative chromosome painting

9.00 Lecture 21: I. King (Aberystwyth, UK) Introgression mapping: a tool to dissect the grass genome

9.30 Lecture 22: J.H. de Jong (Wageningen, The Netherlands) High resolution FISH reveals the molecular and chromosomal organisation of repetitive sequences in tomato

10.00 Coffee

10.30 Lecture 23: K. Fukui (Niigata, Japan) Smallness: gain and loss in chromosome research

11.00 Lecture 24: I. Chudoba (Metasystems, Jena, Germany) High resolution multicolor banding of human chromosome 5

11.30 Closing lecture: H.C. Macgregor (Leicester, UK) The future of chromosomes

12.00 Plenary session and venue of the 14th ICC

12.15 Closing ceremony

13.00 Lunch

Afternoon free

20.30 Banquet; Fireworks; Disco 